\title{
El ejercicio de la ciudadanía en época de la pandemia COVID- 19 en la ciudad de Arequipa
}

\author{
The exercise of citizenship at the time of the COVID-19 in the city of Arequipa
}

\author{
Alison Serruto Castillo (D), Alexis Francisco Vizcarra Tacca (D), Víctor Alfonso Rivera \\ Flores (D), Fiorela Luz Sana Chalco (D)
}

\section{Cómo citar}

Serruto Castillo, A., Rivera Flores, V. A., Vizcarra Tacca, A. F., y Sana Chalco, F. L. (2021). El ejercicio de la ciudadanía en época de la pandemia COVID-19 en la ciudad de Arequipa. Socialium, 5(1), 37-55. https://doi.org/10.26490/uncp.sl.2021.5.1.761

${ }^{1}$ Doctora en Ciencias Sociales, Socióloga, Universidad Católica Santa María, Arequipa, Perú. aserruto@ucsm.edu.pe Google Scholar

2 Licenciado en Antropología, Educador social, Universidad Nacional de San Agustín de Arequipa, Arequipa, Perú. alexisvizcarra1305@gmail.com Google Scholar

${ }^{3}$ Doctor en Ciencias Sociales, Doctor en Derecho, Magister en Ciencias: Educación Superior, Licenciado en Educación Especialidad en Ciencias Sociales, Abogado,

Universidad Católica Santa María, Arequipa, Perú. vriveraf@ucsm.edu.pe Google Scholar

${ }^{4}$ Licenciada en Sociología, Universidad Nacional de San Agustín de Arequipa, Arequipa, Perú.

nac@unsa.edu.pe Google Scholar

Arbitrado por pares ciegos Recibido: $30 / 10 / 2020$

Aceptado: 24/12/2020

\section{RESUMEN}

El estudio tiene como objetivo conocer el impacto del estado de emergencia en el ejercicio de los derechos ciudadanos durante la pandemia Covid - 19 en el periodo de marzo a noviembre del 2020 en la ciudad de Arequipa. La investigación utilizó una metodología mixta, haciendo uso de técnicas, tales como la revisión documental y una encuesta virtual a los ciudadanos Arequipeños mayores de 18 años, de tipo básico, nivel descriptivo, diseño no experimental de corte transversal. En lo que respecta a la muestra, se encuesto a un total de 384 personas. Entre los resultados más relevantes se muestra que los derechos restringidos que han generado mayor malestar en la población son los referidos a la libertad de reunión, libertad de tránsito y el derecho al trabajo. Y entre los derechos vulnerados no restringidos se encuentra los derechos de los niños y el de la igualdad entre individuos.

Palabras clave: pandemia; COVID-19; ciudadanía; derechos; estado de emergencia.

\section{ABSTRACT}

The study aims to know the impact of the state of emergency on the exercise of citizen rights during the COVID-19 pandemic in the period from March to November 2020 in the Arequipa region. The research used a mixed methodology, making use of techniques such as documentary review and a virtual survey of Arequipeños citizens over 18 years of age, basic type, descriptive level, nonexperimental cross-sectional design. Regarding the sample, a total of 384 people were surveyed. Among the most relevant results, it is shown that the restricted rights that have generated the greatest discomfort in the population are those related to freedom of assembly, freedom of movement and the right to work. And among the unrestricted violated rights are the rights of the child and that of equality between individuals.

Keywords: pandemic; COVID-19; citizenship; rights; state of emergency. 


\section{Introducción}

El desarrollo de las distintas sociedades desde sus inicios implicó la instauración de un régimen social basado en el derecho como señala Castro (2014), "desde que el ser humano se arraigó sobre la tierra han existido instituciones. Al agruparse en asociaciones y sociedades, los individuos fueron creando órdenes jurídicos que regularían y conciliarían sus relaciones, es decir, fueron creando derechos" (p.17).

Estas instituciones están representadas por un hombre (Platón, 1965) a quien se le da el encardo encargo de dictar y hacer respetar nuestras leyes. Este hombre es, en nuestros tiempos modernos el presidente de la republica que es elegido por voto popular en los países cuyo régimen político proviene del derecho occidental. La autoridad que sustenta el presidente emana de la voluntad popular pero su accionar está supeditado a las leyes de la nación que gobierna. Así, en nuestro país su poder debe estar basado en la constitución política que reúne las leyes que los representantes del pueblo trascribieron por ser de origen popular su concepción, siendo dichas leyes el reflejo del bienestar que el pueblo desea y exige. Durante la pandemia COVID - 19 el estado peruano representado por el presidente Martín Vizcarra Cornejo aplicó una serie de medidas restrictivas a la población para disminuir el índice de contagios y evitar que colapse el sistema de salud. Estas medidas fueron sustentadas en los artículos $7^{\circ}$ y $9^{\circ}$ de la constitución política del Perú que dan potestad al gobierno de proteger la salud de la nación y su difusión. Las restricciones emitidas durante el estado de emergencia en nuestro país dieron a conocer las diferencias entre un porcentaje poblacional que podían laborar por ser consideradas sus actividades como esenciales en contraposición de la mayoría poblacional que tuvo que acatar el estado de inmovilización obligatoria por no ser considerada su actividad laboral como no esencial, pero ¿Cuáles fueron los criterios para considerar que actividad es o no esencial?, cacaso el derecho a laborar por la necesidad de alimentación no es esencial?, ¿Qué medidas se tomaron para enfrentar la pandemia en el Perú?, ¿Cómo reacciono la población arequipeña ante las medidas implementadas por el gobierno?, ¿Cuáles son los derechos ciudadanos que se han afectado con la implementación de las medidas sanitarias a nivel nacional para frenar la pandemia COVID-19.

Para dar respuesta a estas interrogantes se ha realizado el presente estudio a la luz de los artículos de la constitución política del Perú que fueron usados para limitar el actuar de muchos y permitir el de pocos, además se aplicó una encuesta para conocer el sentir del poblador de la ciudad Arequipa en relación al presente tema. 
En tanto, revisando como parte de los antecedentes del tema, el estudio del Instituto de Democracia y Derechos Humanos (2020) menciona que el Comité de Derechos Económicos, Sociales y Culturales (DESC), la Comisión Interamericana de Derechos Humanos (CIDH) y la Corte la corte Interamericana de Derechos Humanos (Corte IDH) coinciden en el impacto que tiene el COVID-19 sobre los derechos económicos, sociales y culturales.

De esta manera el CIDH recomienda que los Estados deben notificar a las partes correspondientes de su decisión de suspender ciertos derechos, asegurarse de que esta decisión no tenga efectos discriminatorios, y abstenerse de suspender derechos inderogables, como el derecho a la vida o la prohibición de tortura, tratos crueles, inhumanos y degradantes (IDEHPUCP, 2020).

En este sentido, (Human Rights Watch, 2020) argumenta que las cuarentenas generales y los confinamientos de duración indeterminada rara vez cumplen estos criterios y, a menudo, se imponen precipitadamente, sin garantizar la protección de las personas en aislamiento, especialmente las poblaciones vulnerables. Debido a que tales cuarentenas y confinamientos son difíciles de imponer y aplicar de manera uniforme, a menudo su aplicación es arbitraria o discriminatoria.

Al respecto, Amnistía Internacional (2020) explica que la pandemia COVID-19 tiene el potencial de afectar a los derechos humanos de millones de personas, siendo el derecho a la salud uno de los primeros afectados, seguido de la censura, acoso e intimidación de activistas, la discriminación y xenofobia, detenciones arbitrarias entre otros.

Como lo expresa, Varennes, relator Especial de las Naciones Unidas para las Minorías, mencionó que "El COVID-19 no es solo un problema sanitario, también puede ser un virus que agrave la xenofobia, el odio y la exclusión. Existen informes acerca de la violencia física que sufren los chinos y las personas asiáticas; de los discursos de odio que culpan a minorías, como a los romaníes y a los hispanos, de la propagación del virus; y de políticos que piden que no se permita el acceso de los inmigrantes a los servicios médicos. Todo muestra que los estados deben enfatizar de forma urgente que los derechos humanos de todos, particularmente de los más vulnerables y marginados, deben ser protegidos" (Naciones Unidas, 2020).

\section{Método}

La presente investigación utilizó una metodología mixta (cuantitativa-cualitativa), haciendo uso de las técnicas como la encuesta y la revisión documental, para lo cual aplico un cuestionario online estructurado cerrado, fichas bibliográficas y análisis de contenido, instrumentos claves para la 
recolección y análisis de información. Además, es de tipo básica, es decir que su finalidad es generar nuevos conocimientos, de nivel descriptivo, con un diseño no-experimental y de corte transversal.

\section{Procedimiento}

Para la recolección de información documental se utilizó fichas bibliográficas con el fin de revisar toda la información referente a las medidas sanitarias para luego pasar al análisis de contenido de la información recopilada. Después se realizó la identificación de los derechos restringidos de la constitución política del Perú y la ley general de salud que fueron utilizadas como parte del procedimiento del gobierno para limitar las acciones de la población a nivel nacional.

En cuanto a la recolección de información de la población Arequipeña se utilizó como instrumento un cuestionario virtual estructurado cerrado con el fin de mostrar la valoración poblacional sobre la aplicación de las medidas adoptadas por el gobierno, logrando de esta manera vincular la actividad teórica con la empírica en aras del conocimiento social.

Para la aplicación del cuestionario se consideró como población universo a todas las personas que residen actualmente en la ciudad de Arequipa conformada por 21 distritos (Arequipa (Cercado), Alto Selva Alegre, Cayma, Cerro Colorado, Characato, Chiguata, Jacobo Hunter, José Luis Bustamante y Rivero, Mariano Melgar, Miraflores, Mollebaya, Paucarpata, Sabandía, Sachaca, Socabaya, Tiabaya, Uchumayo, Yanahuara, Yura, Quequeña y Yarabamba), los cuales suman un total de 1117284 habitantes al presente año, para realizar la estimación se tomó como base los datos del censo nacional 2017 del INEI (Instituto Nacional de Estadística e Informática), considerando un crecimiento anual del $2.8 \%$.

Finalmente, para determinar la muestra aleatoria se aplicó la siguiente formula, de donde se obtuvo la cantidad representativa de 384 encuestados, teniendo un 5\% como margen de error y un $95 \%$ de nivel de confianza.

$$
\begin{gathered}
\mathrm{n}=\frac{N * z^{2} * \alpha^{2}}{(N-1) * e^{2}+z^{2} * \alpha^{2}} \\
\mathrm{n}=\frac{117284 * 1,96^{2} * 0,5^{2}}{(1117284-1) * 0,05^{2}+1,96^{2} * 0,5^{2}} \\
\mathrm{n}=\frac{1073039,5536}{2794,1679} \\
\mathrm{n}=384
\end{gathered}
$$

Considerando lo siguiente: 
$\alpha=$ Valor del nivel de confianza $(95,0 \%)$

$e=$ Margen de error $(0,05)$

$\mathrm{N}=$ Tamaño Población (1117284)

$\mathrm{n}=$ Muestra

\section{Resultado}

\section{Estado de emergencia y la pandemia COVID- 19}

El estado de emergencia es un estado de excepción que permite la restricción de ciertos derechos. De acuerdo a la Constitución peruana, durante los estados de emergencia pueden restringirse o suspenderse los derechos relativos a la libertad y la seguridad personales, la inviolabilidad del domicilio, y la libertad de reunión y de tránsito. Ante estas restricciones es posible interponer garantías constitucionales a fin de examinar su razonabilidad y proporcionalidad. Además, el Perú ha ratificado tratados de derechos humanos que complementan la disposición constitucional, estableciendo un catálogo de derechos que no admiten restricción (PUCP, 2020)

\section{Los derechos ciudadanos en el Perú y la pandemia COVID-19}

El Consejo Interamericano de Derechos Humanos (2020) explica que la pandemia del COVID-19 puede afectar gravemente la plena vigencia de los derechos humanos de la población en virtud de los serios riesgos para la vida, salud e integridad personal que supone el COVID-19; así como sus impactos sobre las sociedades en general.

\section{Medidas implementadas para enfrentar la pandemia COVID-19 en el Perú}

Acorde al Parlamento Andino (2020) se resalta algunas de las medidas implementadas por el gobierno peruano durante la pandemia 2020.

\section{a) Sector Salud}

- Resolución Ministerial Nº39-2020-MINSA del 31 de enero de 2020: aprueba el Documento Técnico: "Plan Nacional de Preparación y Respuesta frente al riesgo de introducción del Coronavirus", para que los sistemas de vigilancia, contención y respuesta sean fortalecidos para enfrentar el COVID-19 en el país.

- Decreto de Urgencia $N^{\circ}$ 037-2020 del 12 de abril de 2020: establece acciones complementarias para el sector salud como la ampliación para otorgar el seguro de vida para todo el personal de la salud. Aprobación del pago de una bonificación extraordinaria de 720 soles para el personal de la salud. 
- Afiliación virtual al Seguro Integral de Salud (SIS) para la población que no cuenta con este beneficio y poder garantizar su atención frente a la pandemia.

- Aprobación del Decreto de Urgencia 066, que establece el oxígeno como un bien esencial y reduce las especificaciones de $99 \%$ a $93 \%$, con el fin de aumentar el mercado. Además, instituye la contratación nacional de requerimientos para la fabricación de oxígeno local, balones, concentradoras y redes para hospitales.

- Centros de Atención Rápida Temporal en los distritos con mayor nivel de contagios de coronavirus, para facilitar el acceso a tamizajes bioseguros, diagnóstico precoz e inicio temprano de procedimientos clínicos en el primer nivel de atención.

\section{b) Sector Económico}

- Decreto de Urgencia № 029-2020: establece acciones para financiar a la micro y pequeña empresa y reducir los impactos que pueda generar la pandemia sobre la economía del país. De igual forma, se creó el Fondo de Apoyo Empresarial a la MYPE (FAE-MYPE) para garantizar los créditos de capital de trabajo a las MYPE, así como reestructurar y refinanciar sus deudas, hasta por 300.000 .000 de soles.

- Decreto Supremo $N^{\circ}$ 011-2020-TR del 21 de abril de 2020: determina acciones complementarias para la aplicación del Decreto de Urgencia $N^{\circ}$ 038-2020, con el propósito de reducir los efectos económicos causados a los trabajadores y empleadores por la pandemia en el país, así como resguardar los empleos.

- Decreto de Urgencia N049-2020 del 27 de abril de 2020: para fortalecer la gestión del Fondo de Apoyo Empresarial a la MYPE (FAE-MYPE), se establecen acciones económicas y financieras, a través de la asignación de nuevos recursos, para que los créditos sean destinados de manera exclusiva para capital de trabajo, así como establece otras acciones para reducir el impacto de la pandemia en la economía.

- Aprobación de una disposición que faculta al Ministerio de la Producción, al Ministerio de Defensa, al Ministerio del Interior y a los gobiernos locales para trabajar conjuntamente con los grupos comerciales y productivos de mypes en la construcción de los protocolos de inicio de actividades y la ejecución de plataformas digitales.

\section{c) Sector Social}

- Asignación de un bono para hogares en condición de pobreza o pobreza extrema correspondiente a 380 soles, así como, un bono de 380 soles para los trabajadores 
independientes, cuyos hogares están calificados como no pobres por el Sistema de Focalización de Hogares (SISFOH).

- La atención de pagos del Programa Pensión 65, que otorga una subvención económica a los adultos mayores seguirá funcionando a nivel nacional.

- En el marco del Estado de Emergencia, alrededor de 16 millones de peruanos serán beneficiarios del fraccionamiento de los recibos de agua, siempre y cuando el consumo sea menor a los 50.000 litros por mes.

- Se aprobó el Bono de Electricidad por un valor de 160 soles, que favorecerá a más de 5 millones de hogares. Mediante este, el Estado pagará directamente a las empresas eléctricas el consumo de dicho servicio.

\section{d) Sector Político}

- A través del Decreto Supremo $\mathrm{N}^{\circ}$ 008-2020-SA del 11 de marzo de 2020 se declaró Emergencia Sanitaria a nivel nacional, por el plazo de noventa (90) días calendario. Diferentes sectores en el ámbito laboral, social, de transporte aéreo, marítimo, fluvial, terrestre, así como los gobiernos locales, entre otros, deben tomar las medidas necesarias para prevenir la propagación del COVID-19 en el país.

- Decreto de Urgencia N ${ }^{\circ}$ 025-2020 del 11 de marzo de 2020: determina medidas urgentes para reforzar el sistema de vigilancia y la respuesta sanitaria ante el riesgo de la propagación del COVID-19, protegiendo la salud de la población y disminuir el impacto sanitario.

- Se aprobó el Decreto Supremo N 139-2020-PCM, que modifica el Decreto Supremo $\mathrm{N}^{\circ} 116-2020-\mathrm{PCM}$, que establece las medidas que debe observar la ciudadanía en la nueva convivencia social y prorroga el Estado de Emergencia Nacional por las graves circunstancias que afectan la vida de la Nación a consecuencia de COVID-19, modificado por los Decretos Supremos N 129-2020PCM y N 135-2020-PCM. Además, este decreto prohíbe cualquier reunión entre familiares o amigos, con el fin de evitar el incremento de los casos de coronavirus.

\section{Derechos ciudadanos afectados por la pandemia COVID-19}

El CIDH (Comisión Interamericana de Derechos Humanos) ha observado que se han suspendido y restringido algunos derechos dentro de los estados de emergencia, entre los derechos vulnerados se resaltan los derechos de la libertad de expresión, el derecho de acceso a la información pública, la 
libertad personal, la inviolabilidad del domicilio, el derecho a la propiedad privada; y se ha recurrido al uso de tecnología de vigilancia para rastrear la propagación del coronavirus, y al almacenamiento de datos de forma masiva. Considerando que, si bien existen impactos sobre todos los derechos humanos frente a los diversos contextos ocasionados por la pandemia, especialmente en relación al derecho a la vida, la salud e integridad personal, se ven seriamente afectados el derecho al trabajo, a la seguridad social, a la educación, a la alimentación, al agua y a la vivienda, entre otros.

\section{Derechos de la constitución Política del Perú}

El artículo $7^{\circ}$ de la constitución política del Perú se refiere al derecho a la protección de la salud que todo ciudadano posee por ser inherente a la persona y su deber de contribuir a su promoción y defensa, el artículo $9^{\circ}$ presenta la facultad que posee el estado de dirigir la política nacional de salud siendo responsable de la descentralización y acceso a la salud de la población. Los artículos citados tocan específicamente el tema de la salud como derecho fundamental, la protección de la salud pública y el acceso a esta de la población. Estas premisas no contienen ningún elemento que faculte al gobierno establecer la restricción de derechos fundamentales como los establecidos en los siguientes artículos de la constitución:

Artículo $2^{\circ}$. - Toda persona tiene derecho:

1. A la vida, a su identidad, a su integridad moral, psíquica y física y a su libre desarrollo y bienestar. El concebido es sujeto de derecho en todo cuanto le favorece.

2. A la igualdad ante la ley. Nadie debe ser discriminado por motivo de origen, raza, sexo, idioma, religión, opinión, condición económica o de cualquiera otra índole.

9. A la inviolabilidad del domicilio. Nadie puede ingresar en él ni efectuar investigaciones o registros sin autorización de la persona que lo habita o sin mandato judicial, salvo flagrante delito o muy grave peligro de su perpetración. Las excepciones por motivos de sanidad o de grave riesgo son reguladas por la ley.

11. A elegir su lugar de residencia, a transitar por el territorio nacional y a salir de él y entrar en él, salvo limitaciones por razones de sanidad o por mandato judicial o por aplicación de la ley de extranjería.

15. A trabajar libremente, con sujeción a ley.

Artículo $22^{\circ}$. - El trabajo es un deber y un derecho. Es base del bienestar social y un medio de realización de la persona. 
Restringir solo algunas actividades laborales de la población durante el estado de emergencia se opone al derecho a la vida (es necesario trabajar para poder generar ingresos para vivir), a la igualdad ante la ley puesto que se priorizaron solo algunas actividades económicas consideradas unilateralmente "indispensables" por el gobierno contraponiéndose al derecho a trabajar libremente considerando que el trabajo es un derecho y un deber que se basa en que genera el bienestar social. Los numerales 9 y 11 le dan la potestad al gobierno de intervenir el domicilio y limitar el tránsito de las personas y el lugar de residencia por razones de sanidad que fueron efectuados en desmedro de los derechos antes citados.

Artículo $44^{\circ}$. - Son deberes primordiales del Estado: defender la soberanía nacional; garantizar la plena vigencia de los derechos humanos; proteger a la población de las amenazas contra su seguridad; y promover el bienestar general que se fundamenta en la justicia y en el desarrollo integral y equilibrado de la Nación.

Resulta anecdótico que el artículo $44^{\circ}$ haga hincapié en el desarrollo equilibrado de la nación, al ver que las medidas adoptadas por el gobierno no se ciñen a los principios expuestos en este artículo, dado que no se promueve el bienestar general, la justicia, ni el desarrollo integral y equilibrado de la mayoría poblacional al impedirles trabajar, acción que provee de los medios necesarios para su supervivencia. Esta situación se agrava al conocer que el empleo informal en nuestro país representaba el $72.4 \%$ de la población que laboraba (INEI, 2019), este empleo se centraba en actividades agropecuarias, pesca y servicios (transporte, alojamientos, restaurantes y comunicaciones). El caso del empleo formal no varía demasiado en relación a las actividades económicas que se afectaron siendo los servicios la principal actividad económica con un $58 \%$ frente a un $33 \%$ en el caso del empleo informal.

Las actividades que se prohibieron a nivel nacional se centraron en el ámbito de los servicios y transporte por ser de uso masivo en su mayoría, pero actividades como la minería y la televisión que no representan formas de trabajo significativo para la población fueron aprobadas de acuerdo al plan de contingencia del estado. Este plan de contingencia sumió en la pobreza a más de 6.7 millones de peruanos que eran parte de la masa laboral formal.

En el caso de la región Arequipa se estima que 4 de cada 10 trabajadores perdieron su empleo durante la pandemia (INEI, 2020), estas cifras al igual que a nivel nacional se refieren al empleo formal que puede ser seguido mediante los registros que maneja el instituto nacional de estadística e informática (INEI), adoleciendo de información de los trabajadores informales que representan más del $70 \%$ del empleo en nuestro país. El artículo $118^{\circ}$ de nuestra constitución faculta al presidente 
hacer uso de las fuerzas armadas y policía nacional para controlar el orden interno y la seguridad externa de la república, facultad que utilizo para restringir la movilización y por ende el trabajo por el estado de la pandemia COVID- 19 en nuestro país. El uso de estas instituciones armadas se debe restringir a: velar por el orden y la seguridad de la población como dicta la constitución mas no a ir en contra del derecho a la vida que solo puede proseguir mediante el ejercicio del derecho al trabajo.

Artículo $137^{\circ}$ - El Presidente de la República, con acuerdo del Consejo de Ministros, puede decretar, por plazo determinado, en todo el territorio nacional, o en parte de él, y dando cuenta al Congreso o a la Comisión Permanente, los estados de excepción que en este artículo se contemplan:

- Estado de emergencia, en caso de perturbación de la paz o del orden interno, de catástrofe o de graves circunstancias que afecten la vida de la Nación. En esta eventualidad, puede restringirse o suspenderse el ejercicio de los derechos constitucionales relativos a la libertad y la seguridad personales, la inviolabilidad del domicilio, y la libertad de reunión y de tránsito en el territorio.

- El plazo del estado de emergencia no excede de sesenta días. Su prórroga requiere nuevo decreto. En estado de emergencia las Fuerzas Armadas asumen el control del orden interno si así lo dispone el presidente de la República.

\section{Figura 1}

Derechos que se han restringido durante la pandemia del COVID-19 en el Perú

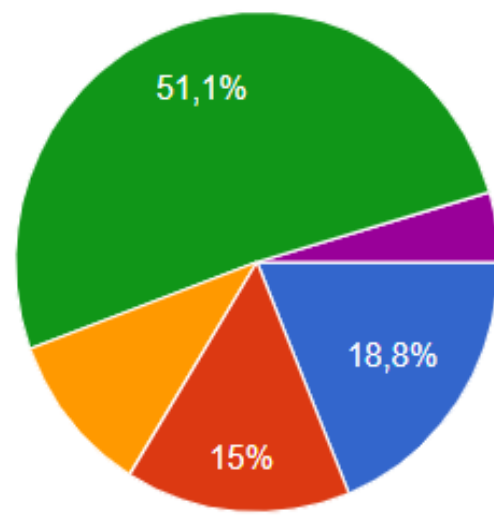

Inviolabilidad del domicilio y la libertad de reunión

A transitar por el territorio nacional y a salir de él y a entrar en él

- A la vida y a trabajar libremente

Todas las anteriores

Ninguna

Nota. Información recogida de la encuesta sobre el Ejercicio de la Ciudadanía en época de la pandemia COVID- 19 (marzo a noviembre del 2020) en la ciudad de Arequipa.

La figura 1 muestra la frecuencia de las respuestas a la interrogante expuesta, apreciándose que un $50,8 \%$ de los encuestados opto por elegir la opción "Todas las anteriores", pues consideran que se han visto afectados todos estos derechos durante la pandemia COVID- 19. Esto significa que cada 
derecho restringido ha tenido impactos significativos en el normal desarrollo de la vida cotidiana de la población encuestada.

\section{Figura 2}

Nivel de Afectación de los Derechos.

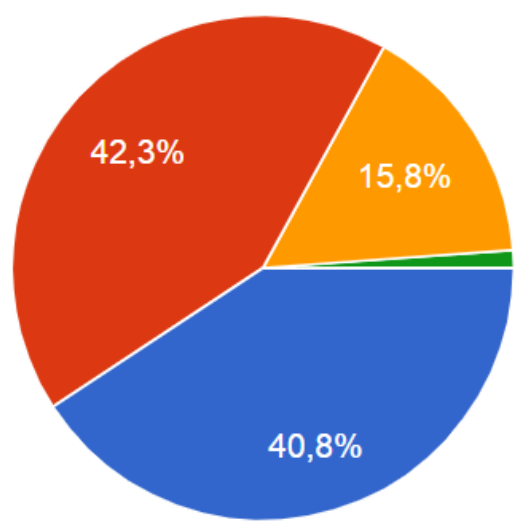

Mucho

Regular

Poco

Nada

Nota. Información recogida de la encuesta sobre el Ejercicio de la Ciudadanía en época de la pandemia COVID- 19 (marzo a noviembre del 2020) en la ciudad de Arequipa.

En la figura 2 se muestra el nivel de afectación de los derechos restringidos durante el estado de emergencia por la pandemia COVID-19, donde se tiene una significativa puntuación negativa, pues el $40,3 \%$ de la población considera haber sido muy afectada, el $42.6 \%$ siente que fue regularmente afectada, el $16 \%$ menciona haber sido poco afectada y solo el $1.1 \%$ considera que no le afecto las restricciones hacia sus derechos.

\section{Figura 3}

Grado de afectación personal de la restricción de derechos

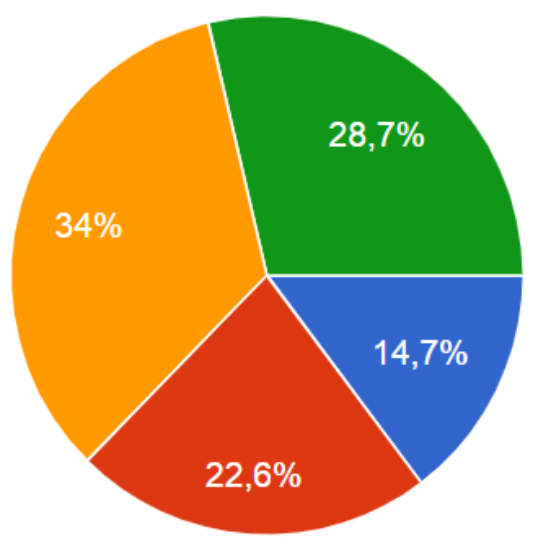

La inviolabilidad del domicilio

La libertad de reunión

La libertad de tránsito

A trabajar 
Nota. Información recogida de la encuesta sobre el Ejercicio de la Ciudadanía en época de la pandemia COVID- 19 (marzo a noviembre del 2020) en la ciudad de Arequipa.

La figura 3 resalta el derecho con mayor afectación personal por parte de los encuestados, entre los derechos a elegir se tienen al derecho a la inviolabilidad del domicilio, la libertad de reunión, la libertad de tránsito y a trabajar. Obteniéndose porcentajes significativos en los derechos a la libertad de tránsito con un $33,5 \%$ y a trabajar con un $28,9 \%$. Por ende, estos derechos son considerados muy importantes en la dinámica familiar y social porque van estrechamente relacionados con la generación de ingresos.

\section{Figura 4}

Apreciación sobre el respeto al derecho del principio de legalidad penal y retroactividad benigna

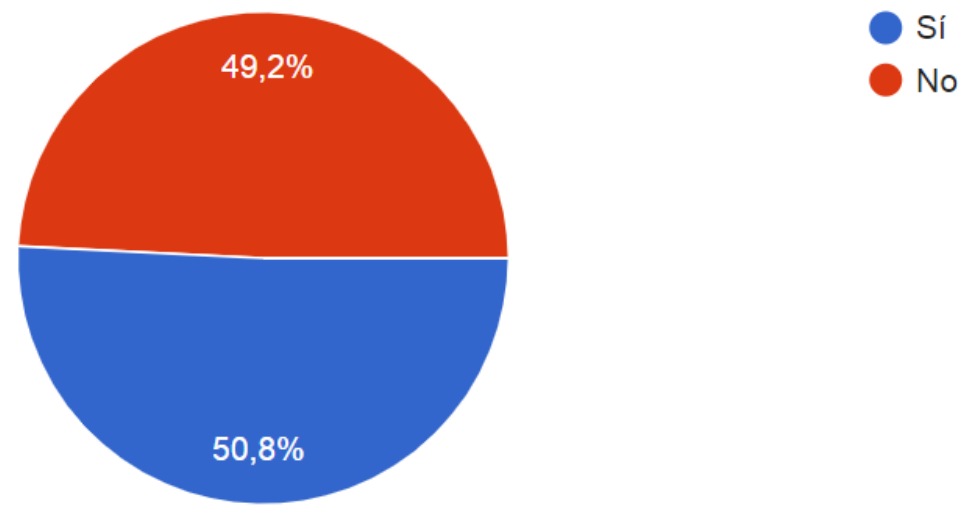

Nota. Información recogida de la encuesta sobre el Ejercicio de la Ciudadanía en época de la pandemia COVID- 19 (marzo a noviembre del 2020) en la ciudad de Arequipa.

El derecho del principio de legalidad penal y retroactividad benigna según el código penal peruano vigente en el artículo II del título preliminar menciona que "nadie será sancionado por un acto no previsto como delito o falta por la ley vigente al momento de su comisión, o sometido a pena o medida de seguridad que no se encuentren establecidas". Acorde a la figura 4 se observa que el 50.8 \% de la población encuestada considera que si se respetó este derecho durante la pandemia, mientras que el $49,2 \%$ opina lo contrario. 


\section{Figura 5}

Apreciación sobre el derecho a la protección de la familia durante la pandemia COVID-19

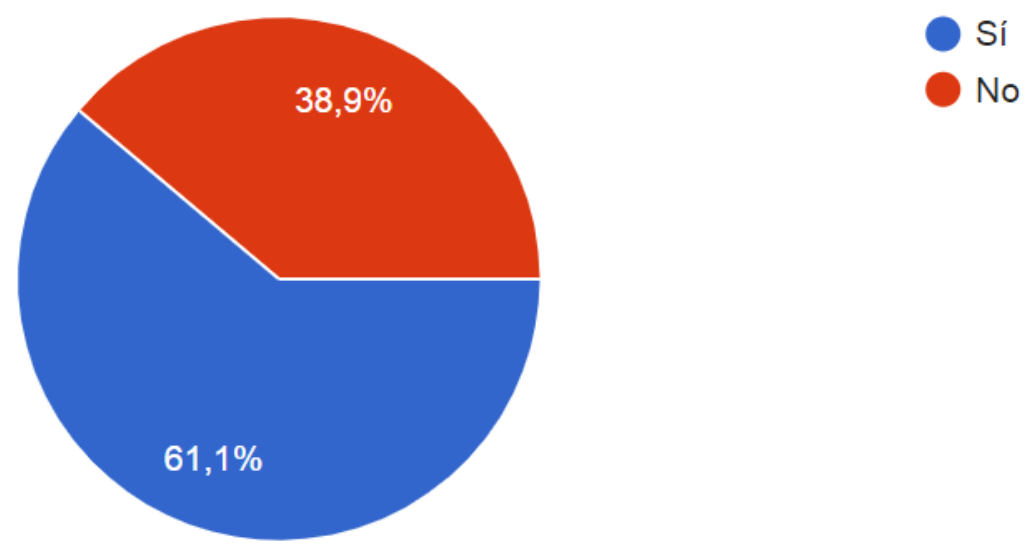

Nota. Información recogida de la encuesta sobre el Ejercicio de la Ciudadanía en época de la pandemia COVID- 19 (marzo a noviembre del 2020) en la ciudad de Arequipa.

Las puntuaciones obtenidas de la población encuestada en torno al derecho de la protección de la familia (ver figura 5) dan como resultado que la mayor parte de los encuestados considera que se vulneró el derecho de la protección de la familia que es una institución fundamental de la sociedad y es deber del estado darle protección (Rubio, 1999).

\section{Figura 6}

Considera que durante esta pandemia se han respetado los derechos del niño

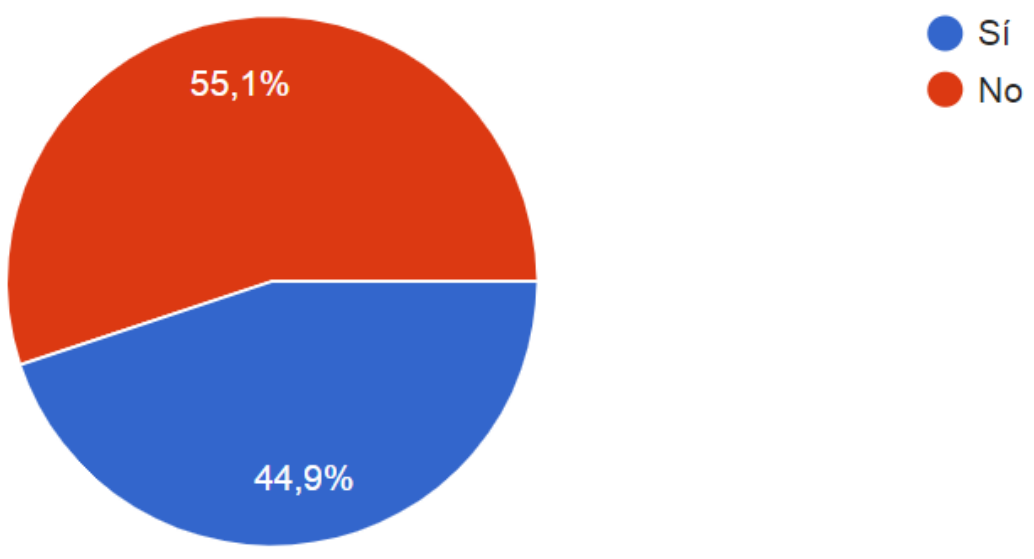

Nota. Información recogida de la encuesta sobre el Ejercicio de la Ciudadanía en época de la pandemia COVID- 19 (marzo a 
noviembre del 2020) en la ciudad de Arequipa.

En cuanto al respeto por los derechos del niño se observa en la figura 6 que el 55,1\% de la población encuestada considera que no se ha respetado estos derechos mientras que el $44,9 \%$ considera lo contrario. De los datos se infiere que las medidas y restricciones durante el estado de emergencia por la pandemia COVID-19 han tenido efectos directamente en la familia, y el aislamiento obligatorio no ha permitido el libre desarrollo de los niños, por ende, la valoración negativa de la población.

\section{Figura 7}

Considera que durante esta pandemia las autoridades han respetado el derecho a la igualdad entre los peruanos.

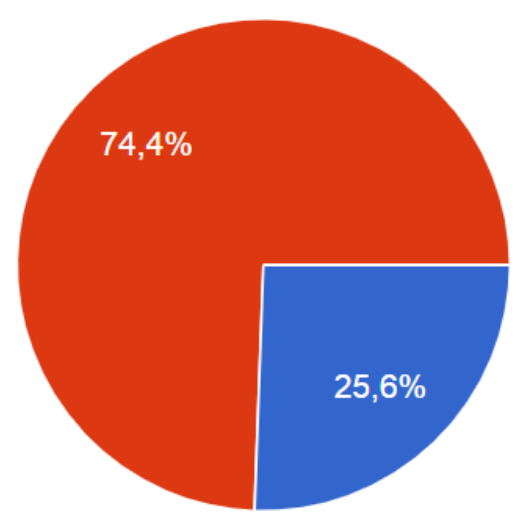

Nota. Información recogida de la encuesta sobre el Ejercicio de la Ciudadanía en época de la pandemia COVID- 19 (marzo a noviembre del 2020) en la ciudad de Arequipa.

La información muestra claramente (ver figura 7) que los encuestados consideran que no se respetó el derecho a la igualdad entre los peruanos $(74,4 \%)$ que nos lleva a abordar el artículo 2 de nuestra constitución política que prohíbe cualquier tipo de discriminación por motivo de origen, raza, sexo, idioma religión, opinión, condición económica o de cualquier índole. 


\section{Figura 8}

Efectos económicos durante la pandemia por las medidas económicas que aplico el gobierno

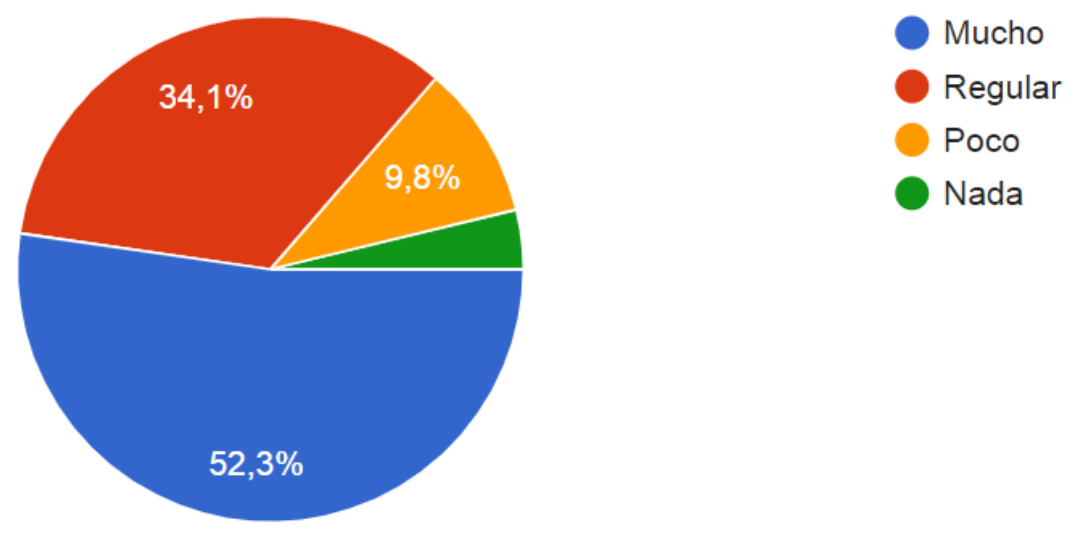

Nota. Información recogida de la encuesta sobre el Ejercicio de la Ciudadanía en época de la pandemia COVID- 19 (marzo a noviembre del 2020) en la ciudad de Arequipa.

Se observa en la figura 8 que más de mitad $(52,3 \%)$ de la población encuestada considera que se vio muy afectada por las medidas económicas que aplico el gobierno. Es necesario considerar que la economía peruana se caracteriza por la informalidad siendo 3 de cada 4 trabajadores parte del sector económico informal situación que revela la falta de capacidad del estado y la empresa privada para crear empleo formal.

\section{Figura 9}

Consideración del Bienestar Familiar por el Gobierno Durante la Pandemia COVID-19

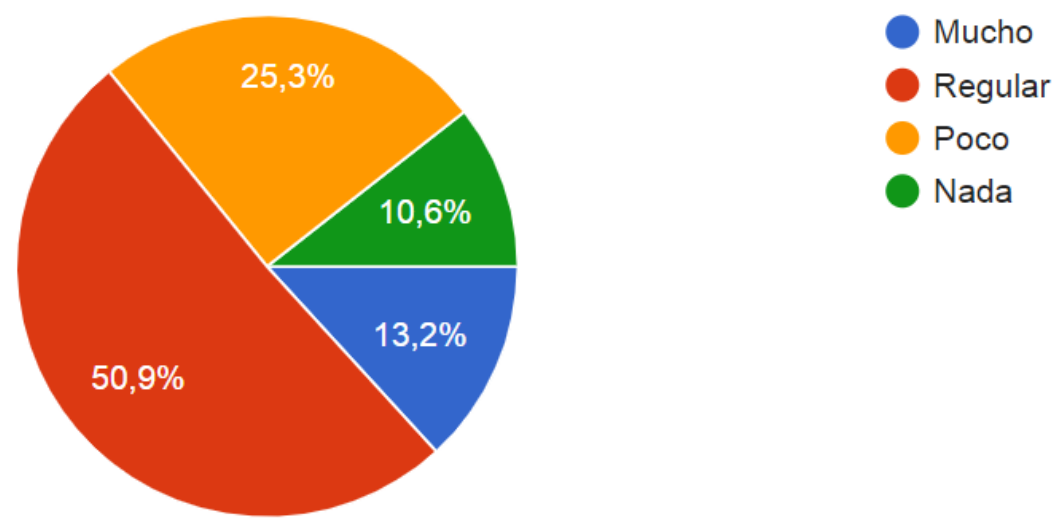

Nota. Información recogida de la encuesta sobre el Ejercicio de la Ciudadanía en época de la pandemia COVID- 19 (marzo a noviembre del 2020) en la ciudad de Arequipa. 
La mitad de la población encuestada $(50,9 \%)$ considera que el gobierno le dio importancia al bienestar de la familia en forma regular, y el $25,3 \%$ lo valora como "poco", solo el $10,6 \%$ lo califica como "nada". Los datos demuestran una actitud intermedia al momento de valorar las acciones del gobierno relacionadas al bienestar de la familia.

\section{Figura 10}

Beneficiarios de bonos económicos durante la pandemia COVID-19

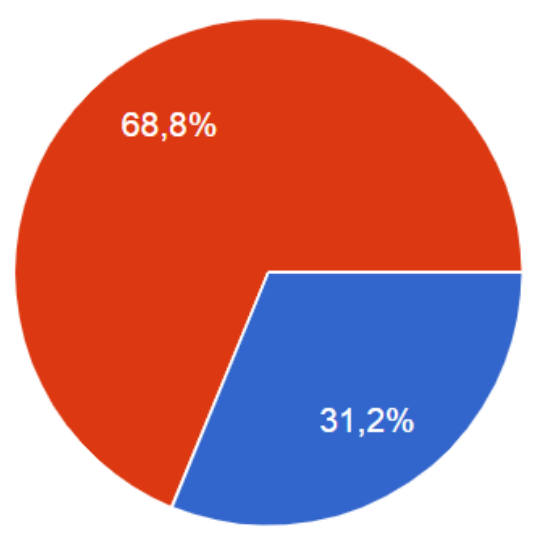

Nota. Información recogida de la encuesta sobre el Ejercicio de la Ciudadanía en época de la pandemia COVID-19 (marzo a noviembre del 2020) en la ciudad de Arequipa.

En cuanto a la recepción de los bonos económicos se observa en la figura 10 que el $68,8 \%$ de la población encuestada fue beneficiaria de este apoyo estatal, mientras que el 31,2\% no recibió tal beneficio, esto significa que la información recopilada es fuente de hogares con cierta vulnerabilidad económica.

\section{Discusión}

Para hacer frente a la enfermedad COVID-19 se ha puesto en marcha medidas que restringen ciertos derechos en las personas, algunas de estas restricciones han tenido grandes impactos en la vida social, especialmente lo referido a lo económico, puesto que nos desenvolvemos en una economía de libre mercado, donde el intercambio monetario por bienes y servicios es constante. Los resultados de la investigación indican que los derechos restringidos que han generado mayor malestar en la población son los referidos a la libertad de reunión, libertad de tránsito y el derecho al trabajo. 
La determinación de actividades económicas esenciales y no esenciales por el decreto supremo № 044 en un país donde el nivel de empleos formales es reducido han dado como resultado el aumento de la pobreza, acorde a la UNICEF (2020) la pobreza se ha incrementado en 10 puntos porcentuales, pasando de $20.2 \%$ a $30.3 \%$ durante la pandemia. Según los datos mostrados en un estudio desarrollado por Grade (2020) a nivel nacional el $74 \%$ de trabajadores de sexo masculino y $82 \%$ del femenino imposibilitados de trabajar a corto plazo son informales, puesto que sus actividades son consideradas no esenciales (Jaramillo \& Ñopo, 2020). Es así que se coincide con los planteamientos de (Human Rights Watch, 2020), quien explica que los efectos de las cuarentenas son diferentes y no se aplican de manera uniforme, y a menudo su aplicación es arbitraria o discriminatoria.

Sin embargo, cabe precisar que durante un estado de emergencia solo pueden restringirse el ejercicio de cuatro derechos humanos, los cuales son el derecho a la libertad y seguridad personales, la inviolabilidad de domicilio, libertad de tránsito y de reunión, pero de ninguna manera se suspenden los derechos intangibles de la persona, entre ellos se encuentran el derecho a la vida, el derecho a la integridad personal, el derecho de conciencia y religión, el derecho a la protección de la familia, el derecho del principio de legalidad penal y retroactividad benigna, el derecho a la integridad personal, los derechos del niño, el derecho a la igualdad, el derecho a las garantías judiciales y a un debido proceso, entre otros (Campos, 2020). En este aspecto, los resultados de investigación evidencian que no se han respetado los derechos referidos a los niños y el de la igualdad entre individuos.

\section{Conclusión}

La restricción de los derechos a la inviolabilidad de domicilio, la libertad de reunión, a transitar por el territorio nacional, a salir y entrar en él, a la vida y a trabajar libremente ha presentado un impacto negativo en la población que se ha visto afectada por primera vez en su historia al restringirse derechos tan elementales, que más allá de su valor intrínseco individual representan por su dimensión un elemento importante de la dinámica socioeconómica de nuestro país que se caracteriza por el autoempleo al no poseer el estado la capacidad de generar fuentes de empleo formal. La autogeneración de empleo por parte de la población determina que cualquier tipo de fluctuación en los derechos mencionados afecten significativamente en el ingreso económico familiar hecho que está sustentado en los resultados obtenidos mediante el instrumento aplicado a la muestra obtenida siendo representativa la información presentada. 
Las medidas subsidiarias (bono familiar universal, bono rural y bono independiente) que implemento el estado para sostener la economía familiar de la población de riesgo que se vio afectada al no poseer ingresos por las restricciones implementadas, no fue relevante por las deficiencias en su distribución y focalización no llegando a abarcar al grueso de la población pobre y pobre extrema que se constata con los resultados de la presente investigación.

\section{Referencias}

Amnistía Internacional. (05 de 02 de 2020). Siete maneras en que el coronavirus afecta a los derechos humanos. Amnesty. https://www.amnesty.org/es/latest/news/2020/02/explainer-sevenways-the-coronavirus-affects-human-rights/

Campos, E. (7 de 08 de 2020). Pasión por el Derecho. ¿Qué derechos se suspenden en estado de emergencia? LP. Derecho. https://Ipderecho.pe/que-derechos-se-suspenden-en-estado-deemergencia/

Campbell, J. (1991). Las máscaras de dios: mitología oriental. Alianza editorial.

Castro, M. (2014). Los puentes entre la antropología y el derecho: orientaciones desde la antropología jurídica. Programa de Antropología Jurídica e Interculturalidad Facultad de Derecho Universidad de Chile.

CERMI. (2020). El Impacto de la Pandemia del Coronavirus en los Derechos Humanos de Las Personas con Discapacidad en España. Comité Español de Representantes de Personas con Discapacidad. https://www.consaludmental.org/publicaciones/Impacto-coronavirusderechos-discapacidad.pdf

Human Rights Watch. (31 de 03 de 2020). Dimensiones de derechos humanos en la respuesta al COVID-19. Human Rights Watch. https://www.hrw.org/es/news/2020/03/31/dimensionesde-derechos-humanos-en-la-respuesta-al-covid-19\#_Toc36462294

IDEHPUCP. (24 de 04 de 2020). Derechos humanos en tiempos de pandemia: Recomendaciones del sistema universal y del sistema interamericano frente al COVID-19: Instituto de Democracia y Derechos Humanos PUCP. https://idehpucp.pucp.edu.pe/notas-informativas/derechoshumanos-en-tiempos-de-pandemia-recomendaciones-del-sistema-universal-y-del-sistemainteramericano-frente-al-covid-19/ 
Instituto Nacional de Estadística e Informática. (2020, 3 de agosto). Comportamiento de los indicadores de mercado laboral a nivel nacional. INEI. http://m.inei.gob.pe/bibliotecavirtual/boletines/condiciones-de-vida/6/

Jaramillo, M. y Ñopo, H. (2020). Impactos de la epidemia del coronavirus en el trabajo de las mujeres en el Perú. Grupo de Análisis para el Desarrollo (GRADE). https://www.grade.org.pe/wpcontent/uploads/GRADEdi106.pdf

Martín, C. (2020). La verdad de la pandemia: Quien ha sido y porqué. Barcelona: Editorial Planeta.

Naciones Unidas. (13 de 04 de 2020). La protección de los derechos humanos durante la crisis del COVID-19. Naciones Unidas. https://www.un.org/es/coronavirus/articles/protegerderechos-humanos-coronavirus

Parlamento Andino. (2020). Principales medidas adoptadas por el gobierno peruano frente a la emergencia provocada por la COVID-19. Parlamento Andino.

Platón, A. (1965). Las leyes. Editorial Iberia.

PUCP. (16 de 03 de 2020). Los derechos delimitados y el estado de emergencia. Instituto de Democracia y Derechos Humanos PUCP. https://idehpucp.pucp.edu.pe/notasinformativas/los-derechos-delimitados-y-el-estado-de-emergencia/

Rubio, M. (1999). Estudio de la constitución política de 1993. Fondo editorial de la Pontificia Universidad Católica del Perú.

\section{Contribución de los autores}

ASC:Conceptualización , análisis formal, investigación , metodología , redacción: revisión y edición. AFVT:Conceptualización , análisis formal, investigación, metodología, redacción: borrador original. VARF:Conceptualización , análisis formal, investigación, metodología, redacción: borrador original. FLSC:Conceptualización, análisis formal, investigación, metodología, redacción: borrador original.

Fuentes de financiamiento.

Los recursos para viabilizar la presente investigación fueron proporcionados en su integridad por los investigadores responsables de la presente publicación.

\section{Conflictos de interés}

Para la elaboración del trabajo no se presentaron conflictos de interés en ninguno de los autores.

\section{Autor de correspondencia}

aserruto@ucsm.edu.pe 